\title{
El proyecto de vida como reto educativo: Biografía del Desarrollo Integral del estudiante de Educación de Básica Superior
}

\section{The life project as an educational challenge: Biography of the Integral Development of the student of Higher Basic Education}

\author{
Lic. Ericka Figueroa Martínez, Mgs ${ }^{1}$ \\ ericka.figueroama@ug.edu.ec \\ Econ. Pedro Iglesias Mora, $\mathrm{MSc}^{2}$ \\ Ing. Merle Iglesias Mora, $\mathrm{MSc}^{3}$
}

Recibido: 1/09/2016, Aceptado: 1/11/2016

\begin{abstract}
RESUMEN
El trabajo se enfoca en la revisión bibliográfica del docente de Educación Básica Superior, donde se muestra la ausencia de los manifiestos del Proyecto de Vida, su carente presentación en el escenario educativo y el enlace del Proyecto Educativo Institucional, lo cual permite indicar la existencia de este vacío, esto se demuestra en las pocas publicaciones del tema y por eso se justifica esta investigación; la revisión bibliográfica dentro de los métodos científicos utilizados permite al estudiante de Cuarto Semestre de la Carrera de Educación Básica inferir en su ausencia y los pocos documentos encontrados ponen de manifiesto la ínfima validez que se toma como resultado de un proceso lógico y métrico del estudiantado que es tutorado; la metodología es la observación directa a través de un focus group (12 estudiantes) del aula de clase que participaron en un proyecto áulico, vivenciando con la ejemplificación in situ en la escuelas de la zona 8 Guayaquil, Duran y Samborondón. El Proyecto de Vida presentado tiene una perspectiva de tipo personal, cultural y de desarrollo humano; este cuestionario contiene preguntas sencillas y particulares, el cual permite la interacción entre el docente tutor y el estudiante de Educación Básica Superior a través de un enfoque sistémico contemporáneo. Es necesario que el docente - tutor conozca biográficamente a sus estudiantes para que este a su vez potencialice su desarrollo integral y perfeccione el desarrollo humano en cada uno de ellos para su posterior integración social.
\end{abstract}

Palabras clave: Desarrollo integral, Proyecto de Vida, Proyecto Educativo Institucional

\footnotetext{
${ }^{1}$ Gestora de Formación Pedagógica y Ambientes de Aprendizajes. Facultad de Filosofía, Letras y Ciencias de la Educación. Ecuador

2 Docente de la Universidad Tecnológica Empresarial de Guayaquil. Ecuador

3 Docente de la Universidad Tecnológica Empresarial de Guayaquil. Ecuador
} 


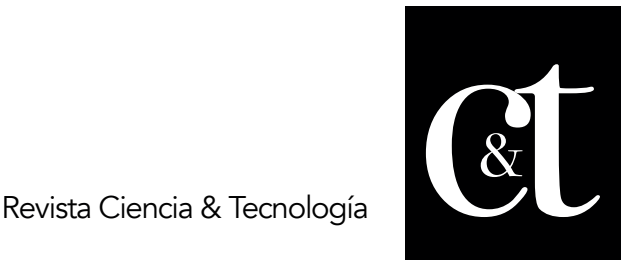

No. 13, 31 de enero de 2017

ISSN impreso: 1390 - 6321

\begin{abstract}
The work focuses on the bibliographic review of the teacher of Higher Education, which shows the absence of manifests of the Life Project, its lack of presentation in the educational scenario and the link of the Institutional Educational Project, which allows to indicate the existence of This void, this is demonstrated in the few publications of the subject and for that reason this investigation is justified; The bibliographic review within the scientific methods used allows the student of the Fourth Semester of the Basic Education Career To infer in its absence and the few documents found reveal the very small validity that is taken as a result of a logical and metric process of the student body that is tutored; The methodology is the direct observation through a focus group (12 students) of the classroom that participated in an aulic project, living with the exemplification in situ in the schools of zone 8 Guayaquil, Duran and Samborondón. he Life Project presented has a personal, cultural and human development perspective; This questionnaire contains simple and particular questions, which allows the interaction between the teacher-tutor and the student of Higher Education through a contemporary systemic approach. It is necessary that the teacher - teacher biographically learns his students so that this in turn potentiates their integral development and perfects the human development in each one of them for their later social integration.
\end{abstract}

Keyword: Integral development, Life Project, Institutional Educational Project

\title{
Introducción
}

Los docentes deben reconocer la importancia del Proyecto de Vida de sus estudiantes de Educación Básica Superior como biografía de sus percepciones y perfiles académicos, reconociendo sus fortalezas y debilidades, al ser esta una semblanza de sus tutoriados, la misma que permite al docente -tutor promover esas fortalezas y disminuir sus debilidades, como parte de su formación docente, involucrará a la familia y traerá a la palestra del claustro educativo la ralea. (Gajardo, 1999) "Las escuelas están en crisis y también lo están las personas que se encuentran a cargo de ellas. Las escuelas han perdido su horizonte hasta hace poco, no cuestionando el título de legitimadores de la educación" (pág. 32), la reflexión está en los docentes, los cuales creen que al ser portadores del conocimiento ya es suficiente para enfrentar el gran reto de formar la nueva generación, sin tener en cuenta la importancia esencia de los valores que deben desarrollar en cada uno de ellos, el currículo oculto no ha muerto sino que ha transmutado a la transversalidad del mismo dentro de cada cátedra; los valores que se han desubicados en el seno del hogar; producto de la migración, familias disfuncionales y una serie de aditivos que desunen la palabra nuclear llamada: familia y esta es la base de todo ser humano para su posterior comportamiento social al no estar fomentada se debe trabajar directamente en las escuelas para recuperar lo que esta no solidifico en su momento y es aquí donde se intensifica la labor del tutor con el Proyecto de Vida a aplicar.

La escuela debe ser el escenario propicio para desarrollar el Proyecto de Vida que a futuro promulgará la empatía no sólo del mundo laboral sino social. (Noro, 2000) "La escuela como ámbito, como estructura edilicia, como espacio común, público y compartido de las actividades educativas se convierte en un escenario en el que los actores institucionales representan diversos papeles..."(pág. 18), es decir, que la escuela a través de los docentes desarrollan estrategias motivadoras de personalidad, 


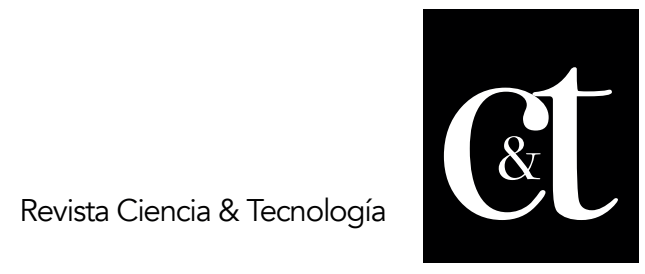

No. 13, 31 de enero de 2017

ISSN impreso: 1390 - 6321

desempeño académico y vinculación en la comunidad que les permita ser ciudadanos del mundo actual capaces de desempeñar su cultura sin vergüenza y prejuicios.

Los estudiantes del Subnivel de Educación Básica y el cual a través de un consenso de Focus Group el cual muestran Hernández, Fernández y Baptista (2014), como ideal para utilizar entre dos grupos de 12 estudiantes para lograr un enfoque efectivo, a través de preguntas claves del tema en las que se destaca la importancia de la biografía estudiantil, sus procesos académicos su perfil y perspectiva laboral en el futuro.

(Senge, 2000) "El dominio personal, es la disciplina que permite aclarar y ahondar continuamente nuestra visión personal, concentrar las energías, desarrollar paciencia y ver la realidad objetivamente" (pág. 25), es una de las propuestas del proyecto áulico que el tutor-docente desarrolle estrategias de conducta y coach para el aprendizaje significativo de la vida.

Vemos al YO (estudiante) como una "organización" con una visión inter, multi y transdisciplinaria de manera integral permitiéndole identificar y comprender con mayor claridad, las múltiples causas y consecuencias de sus acciones a través de una serie de preguntas enfocadas en este modelo.

La elaboración de un proyecto de vida, debe considerar aspectos tales como: el entorno y conocimiento de la persona; la búsqueda de información para satisfacer las inquietudes y posibilidades que los rodean, para alcanzar las metas propuestas; y la flexibilidad, que no debe faltar, pues los seres humanos poseen múltiples intereses, habilidades y la capacidad de rectificar, además los resultados no dependen sólo de la persona. Al definir un proyecto de vida las personas podrán defender lo que piensan, sus intereses y formarse un carácter menos vulnerable al medio. Una de las propuestas en las que se basa el Proyecto de Vida, es la implementación de los valores que representan el espíritu del ideario del PEI, es un documento que claramente identifica el compromiso moral y ético sobre los educandos en el cual reposa el compromiso de hacer de un niño/a un ente integral.

Según Noro (2010), "Las actitudes constituyen un sistema relativamente estable de percepciones y evaluaciones, de sentimientos y emociones, de tendencias a la acción, organizado en relación a una situación significativa o con un objeto propuesto. Engloban elementos perceptivos, interpretativos y valorativos, y una disposición a la acción interior o exterior" (pág. 41).

El proceso del Proyecto de Vida que se presentó a los estudiantes de Educación Básica Superior, es un esquema utilizado en las diferentes instituciones educativas con un gran significado de independencia y creatividad, potenciando construir proyectos en los que el hombre despliega sus aptitudes y recursos, anticipa un futuro en el que la meta de la autorrealización despierta y sostiene ese despliegue, dichos proyectos giran de modo especialmente destacado en torno de dos grandes cuestiones, el amor y el trabajo.

Para que el hombre pueda construir su proyecto de vida, debe cumplir con ciertas condiciones básicas, de tal suerte que asegure la conservación y el desarrollo de sus potencialidades. Esas condiciones surgen de la interacción hombre-mundo, desde su 


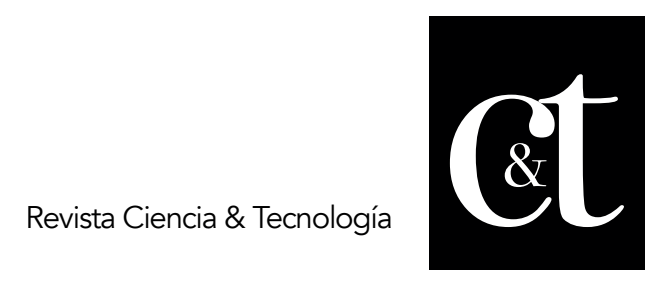

No. 13, 31 de enero de 2017

ISSN impreso: 1390 - 6321

hogar, siendo el docente el que cambie la cosmogonía del estudiante siendo la escuela un lugar ambientado a la creatividad, pero es más un internalizado de valores.

\section{Desarrollo}

La respuesta Vygostkyana de la situación social del proyecto de vida está enfocada en el uso por parte de los seres humanos de signos, para mediar las relaciones entre las personas. Para clarificar el papel que juega el lenguaje en el surgimiento de actividades cognoscitivas estrictamente humanas, consideremos en mayor detalle dos rasgos esenciales de la secuencia de desarrollo antes descrita: (1) mediación a través de signos, y (2) internalización de estos signos, estos signos están visualizados dentro de esta investigación en su árbol genealógico que debe ser un momento de internacionalización de sus raíces, reconociendo desde donde se vive, por ser un país multiétnico y pluricultural, Ilamado Ecuador. La otra opción dentro de la investigación es la realización de inteligencias por eso la necesidad de la presencia del DECE y luego se realizará un reconocimiento de sus habilidades por parte de su propio testimonio, sin que el tutor desmerezca la capacidad y competencia que tenga el estudiante, más bien indicará en que ámbitos profesionales desarrollará en el mundo laboral esta habilidad, afianzando la confianza en el estudiante.

El proyecto o plan de vida representa, entonces, en su conjunto, "lo que el individuo quiere ser y lo que él va a hacer en determinados momentos de su vida, así como las posibilidades de lograrlo" (D’Angelo, 1986, pág. 67). El proyecto de vida desempeña, una función de integración direccional, valorativa e instrumental, de las orientaciones de la personalidad, con los modos posibles de su realización concreta en la actividad, conservando la unidad de sentido general de toda la personalidad.

Para fomentar un proyecto de vida se basa en la familia y la escuela juega un rold esencial en ellos pues esta enfoca las necesidades del estudiante en función de las exigencias de la sociedad y del medio que los rodea; es por ellos que el estado se preocupa por el área fiscal propone una rendición de cuenta no sólo de tipo legal sino moral, en el caso del Estado Ecuatoriano se caracteriza por la mejora y desarrollo de sus ciudadanos en el área educativo está establecido desde la perspectiva de las unidades del milenio. "El estado asume un rol central en la formación de maestros y la docencia se constituye históricamente como profesión de Estado por decisión del propio Estado en el contexto de la organización nacional y de la integración del país" (Noro 2010, pág. 36).

Los esquemas que ayudan a que la institución educativa mejore un proyecto de vida estudiantil en relación de la necesidad integral de estudiante está basado en la política pública debe estar expuesto de la siguiente manera:

\section{Integración con la comunidad}

Plan Educativo Institucional que contempla una perspectiva estratégica, el cual incorpora articuladamente a los padres de familia, la comunidad y diversos sectores sociales, productivos, culturales, deportivos, entre otros.

\section{Organigrama}

Las escuelas deben desarrollar Proyectos de Vida como parte de su proceso educativo y el correcto funcionamiento de estas etapas debe promoverlo el Directivo, la 
aportación de esta investigación está desarrollada en este cuadro Organigrama Proyecto de Vida que se presenta a continuación:

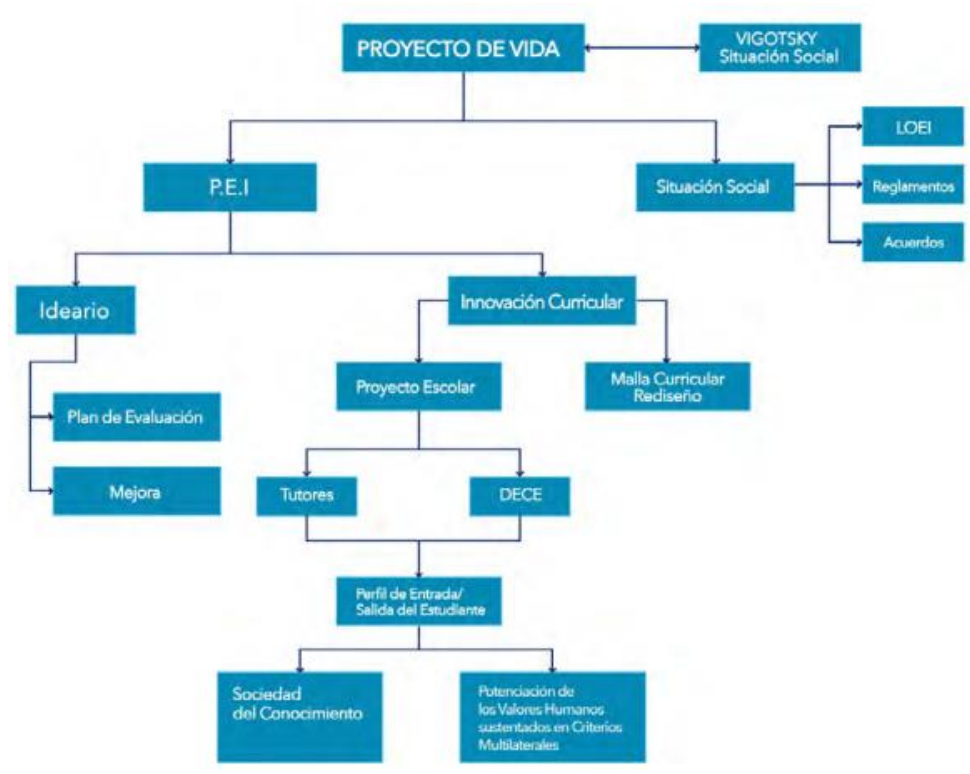

\section{Gráfico 1: Organigrama del Proyecto de Vida}

Fuente: MSc Ericka Figueroa Martínez 1-Docente Universitaria Carrera de Educación Básica

\section{Generalidades del Proyecto de Vida}

Este incluye los elementos más importantes que deseas que haya en tu vida. Es un bosquejo de cómo quiero que sea mi vida, de qué quiero que haya en ella cómo me integraré a la sociedad a partir de este proyecto: relaciones, actividades, personas, lugares, cosas, trabajo, familia.

El punto de partida Mi situación: Mis fortalezas y debilidades. Autobiografía: Se realiza en forma de preguntas sus intereses y la influencia general de personas en su vida, entre otros. Rasgos de mi personalidad: Entre ellos pueden estar los aspectos que no le gustan y los que le gustan como: aspecto físico, relaciones sociales, vida espiritual, vida emocional, aspectos intelectuales y aspectos vocacionales Quién soy: Que impulsa al desarrollo y como influyo en los cambios y ellos en la personalidad, acciones que pudiera tomar, entre otros.

¿Quién seré? Convertir sueños en realidad: Futuros sueños que pueden o no ser alcanzados, las realidades que los favorecen, entre otros Reconoce su identidad intercultural a través del árbol genealógico.

Reconoce sus fortalezas al realizar acciones mejor que otras personas (oficios) Aplicar el Test de las Inteligencias Múltiples de Gardner. 


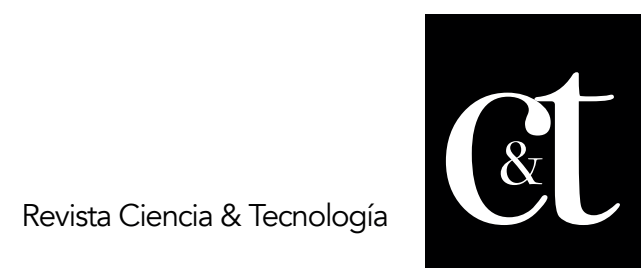

No. 13, 31 de enero de 2017

ISSN impreso: 1390 - 6321
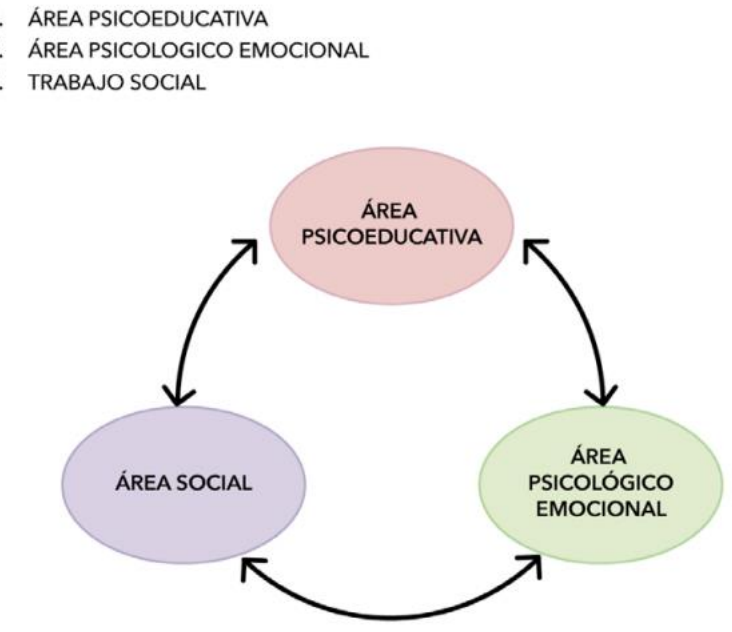

\section{Gráfico 2: La estructura que el manejo del DECE del Ecuador en función del Proyecto de Vida}

Fuente: Elaboración propia

Se cumple con el objetivo planeado de desarrollar competencias de prácticas docentes en estudiantes de la Carrera de Educación Básica a través del estudio y mejora del Proyecto de Vida como biografía estudiantil, a su vez un medio potenciador de lo personal, cultural y de desarrollo humano en estudiantes de Educación Básica Superior de la Zona 8.

En el informe de la UNESCO, menciona que aprender a hacer está relacionado estrechamente con la formación profesional y con la forma como se enseña al alumno a poner en práctica sus conocimientos. En el caso de aprender a ser abarca el despliegue completo del ser humano; además, en relación con el desarrollo personal, nuevamente menciona que "este desarrollo del ser humano, que va del nacimiento al fin de la vida, es un proceso dialéctico que comienza por el conocimiento de sí mismo y se abre después a las relaciones con los demás" (Delors, 1996. pág. 10).

El proyecto áulico desarrollado en la Carrera de Educación Básica a través de un análisis de la situación problémica de educación básica de la Zona 8 (Guayaquil, Durán, Samborondón) demuestra el desconocimiento en los docentes del Proyecto de Vida y su praxis es tomada a menos, sin la responsabilidad necesaria para esta noble misión; después de este diagnóstico de observación directa los estudiantes recolectaron los datos he indicaron en sus resultados que los docentes no reconocen este proyecto de vida como un medio biográfico de contenido oportuno para direccionar a los estudiantes en su desarrollo integral.

Para la obtención de los resultados se llevó a cabo 1 focus group de 12 estudiantes en las instalaciones del Complejo Norte, Cuarto Semestre A1, los cuales fueron entrevistados a través de esta técnica y estos coinciden en la necesidad de que el tutor docente empodere su rol a través del proyecto de vida con una visión integral 
dentro y fuera del aula. La docente justifico este proceso a través de un proyecto áulico que consistió en el desarrollo de la praxis educativa en algunas instituciones de la zona 8.

\section{Tabla 1: Participantes en el focus group}

\begin{tabular}{|c|c|c|}
\hline Edad & Personal Seleccionado & Total \\
\hline $20-25$ & 7 & 7 \\
\hline $25-30$ & 3 & 3 \\
\hline $30-35$ & 2 & 2 \\
\hline Total & 12 & 12 \\
\hline
\end{tabular}

Fuente: Elaboración propia

Universo: Hombres y mujeres de edades comprendidas entre los 20 y 35 años.

Las dinámicas de grupo se realizaron entre los días 25 al 30 de enero, Zona 8 (Guayaquil, Durán y Samborondón).

Las escuelas de las zonas no tenían un programa claro del proceso del Proyecto de Vida, por eso el $90 \%$ de las escuelas visitadas demuestran un desconociendo mientras que el $10 \%$ utilizan el documento como un requisito que existe el ministerio con el acuerdo 242-11.

\section{Conclusiones}

El proyecto de vida y sus relaciones comunes con el devenir educativo de los noveles docentes que se enfocan en la educación básica superior, entre las habilidades y competencias de la praxis educativa deben empoderar su rol de tutores que permite tener una biografía clara de sus educandos.

\section{Recomendaciones}

El Estado deben proponer Proyectos de Vida al MINEDUC1 a sus estudiantes a través de la visión cosmogónica de sus entornos y comunidades porque se debe visualizar a las instituciones por Distritos y Circuitos estas están sectorizaciones geo referenciadas deben tener las consignas educativas y académicas estandarizadas permitiendo la aportación social externa en los proyectos de vida de los estudiantes su propia realidad o contextos sociales; lo constructos deben ser trabajados a través de esta visión responsable de la codificación social del educando e interpretar su costumbres y emociones dentro del contexto educativo.

\section{Referencias Bibliográficas}

D'Angelo Hernández, O. (1986). Proyecto de vida y desarrollo integral humano. Recuperado

http://biblioteca.clacso.edu.ar/ar/libros/cuba/cips/caudales05/Caudales/ARTICUL OS/ArticulosPDF/07D050.pdf 


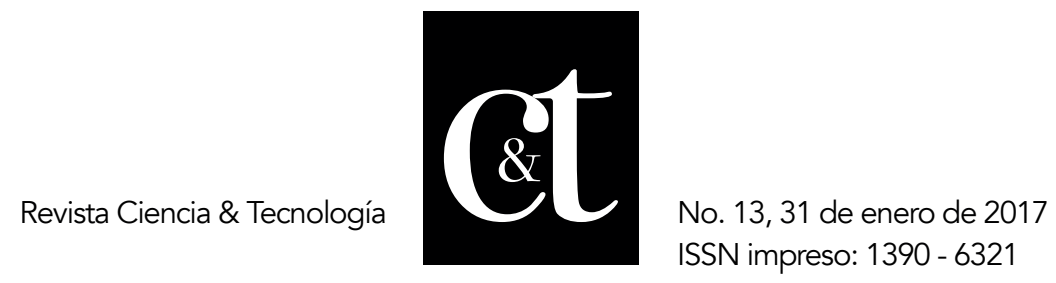

Delors, J. (1996.). Los cuatro pilares de la educación. En: La educación encierra un tesoro. Informe a la UNESCO de la Comisión internacional sobre la educación para el siglo XXI, 91-103, Madrid, España: Santillana/UNESCO.

Gajardo, M. (1999). Reformas Educativas en América Latina: Balance de una Década. Santiago, Chile: Preal/Documentos.

Gajardo, M. (1993). Ivan Illich. Perspectiva Unesco, vol. XXIII, n 3-4, 808-821.

Hernández, Fernández y Baptista (2014). Metodología de la investigación. México: McGraw-Hill.

Noro, J. (2000). Subsidios y estrategias para el crecimiento personal de las instituciones. Rosario Santa Fe: Didascalia.

Noro, J. (2010). Actitudes y valores puerta 1Ministerio de Educación de entrada a una nueva escuela significativa. Revista Iberoamericana de Educación, 4.

Noro, J. (2010). Actitudes y valores puerta de entrada a una escuela nueva significativa. Revista Iberoamericana de Educación, 3.

Noro, J. (s.f.). Biblioteca Docente. Obtenido de Educrea: hhtp;//educrea.cl/formación-de- formadoresdocentes-funcionales-al sistemaointelectuales-criticostransformadores/

Senge, P. M. (2000). La Quinta Disciplina. Recuperado de https://www.academia.edu/33324954/La_quinta_disciplina_Peter_Senge_FREEL IBROS_ORG 